\title{
Lipschitz Invariance of Critical Exponents on Besov Spaces
}

\author{
Qingsong $\mathrm{Gu}^{1, *}$ and Hui $\mathrm{Rao}^{2}$ \\ ${ }^{1}$ Department of Mathematics, The Chinese University of Hong Kong, Hong Kong, \\ China \\ 2 Department of Mathematics and Stochastic, Huazhong Normal University, Wuhan, \\ Hubei 430072, China
}

Received 28 October 2019; Accepted (in revised version) 4 November 2019

Dedicated to Professor Weiyi Su on the occasion of her 80th birthday

\begin{abstract}
In this paper we prove that the critical exponents of Besov spaces on a compact set possessing an Ahlfors regular measure is an invariant under Lipschitz transforms. Under mild conditions, the critical exponent of Besov spaces of certain selfsimilar sets coincides with the walk dimension, which plays an important role in the analysis on fractals. As an application, we show examples having different critical exponents are not Lipschitz equivalent.
\end{abstract}

Key Words: Lipschitz invariant, Besov space, critical exponents, walk dimension, heat kernel.

AMS Subject Classifications: 35K08, 28A80, 35J08, 46E35, 47D07

\section{Introduction}

Let $\left(X, d_{1}\right)$ and $\left(Y, d_{2}\right)$ be two metric spaces. We say that $T:\left(X, d_{1}\right) \rightarrow\left(Y, d_{2}\right)$ is a biLipschitz transform, if $T$ is a bijection and furthermore, there exists a constant $C>0$ such that for any $x, y \in X$,

$$
C^{-1} d_{1}(x, y) \leq d_{2}(T x, T y) \leq C d_{1}(x, y)
$$

The study of Lipschitz equivalence of self-similar sets are initiated by Falconer and Marsh [5] and David and Semmes [4]. Rao, Ruan and Xi [18] (2006) answered a question posed by David and Semmes [4], by showing that the self-similar sets in Fig. 1 are Lipschitz equivalent. After that, there are many works devoted to this topic, for example, $\mathrm{Xi}$ and Xiong [24,25], Luo and Lau [15], Ruan et al. [22], and Rao and Zhang [19]. However, the studies mentioned above are all on self-similar sets with simple topological structure, that is, the fractals under consideration are totally disconnected.

*Corresponding author. Email addresses: 001gqs@163.com (Q. S. Gu), hrao@mail .ccnu . edu.cn (H. Rao) 
Recently, there are some studies on a class of self-similar sets which are not totally disconnected. A non-empty compact set satisfying the set equation $F=\bigcup_{d \in \mathcal{D}} \frac{F+d}{n}$ is called a fractal square if $n \geq 2$ and $\mathcal{D} \subset\{0,1, \cdots, n-1\}^{2}$. Ruan and Wang [21] studied fractal squares of ratio $1 / 3$ and with 7 or 8 branches, which are all connected fractals. Their method is to find various connectivity properties, which depend on very careful observations. Rao and Zhu [20] and Zhu and Yang [26] construct bi-Lipschitz mappings between the fractals illustrated in Fig. 3.

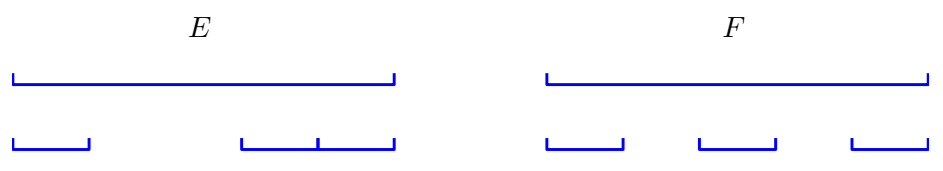

Figure 1: The Cantor sets $E$ and $F$ are Lipschitz equivalence [18].

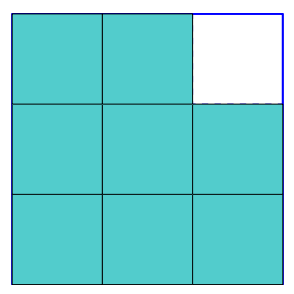

(a)

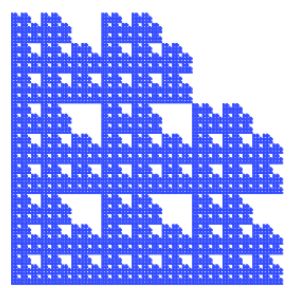

(d)

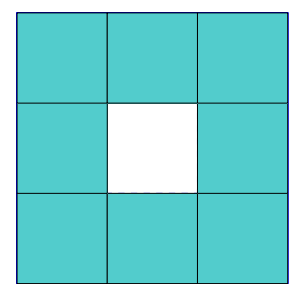

(b)

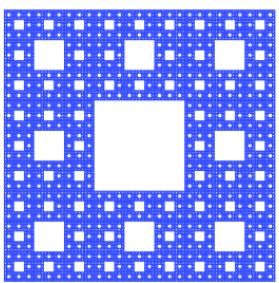

(e)

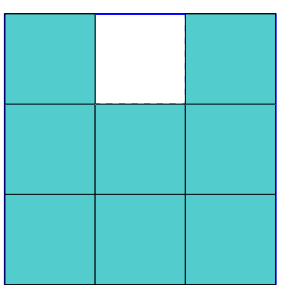

(c)

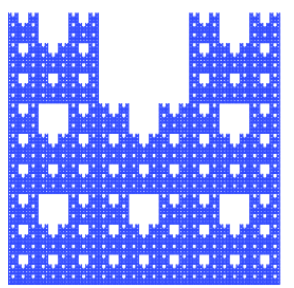

(f)

Figure 2: [21] shows that the above three fractal squares are not Lipschitz equivalent.

The study of the Lipschitz equivalence of connected self-similar sets is a very hard problem. Let us consider the fractals in Fig. 4. By a result of Whyburn [23], they are homeomorphic, and [21] conjectures these two fractal squares are not Lipschitz equivalent (see also [17]). To show two sets are not Lipschitz equivalent, the main method is to construct a certain Lipschitz invariant to distinct them.

In this paper, we use the Besov spaces to construct Lipschitz invariants for fractals 
admitting Ahlfors regular measures.

Let $F$ be a compact subset of $\mathbb{R}^{n}$. A Borel measure $\mu$ on $F$ is said to be Ahlfors regular, if there exists $0<\alpha \leq n$ and a constant $C>0$ such that for any Euclidean metric ball $B(x, r)$ with $0<r<1$,

$$
C^{-1} r^{\alpha} \leq \mu(B(x, r)) \leq C r^{\alpha} .
$$

The set $F$ is called an $\alpha$-set if it admits an Ahlfors regular measure. It is known [11] that such $\mu$ is equivalent to the $\alpha$-dimensional Hausdorff measure on $F$; moreover, if $F$ is a self-similar set satisfying the open set condition, then $F$ is an $\alpha$-set.

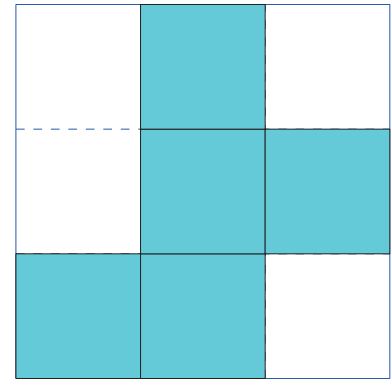

(a)

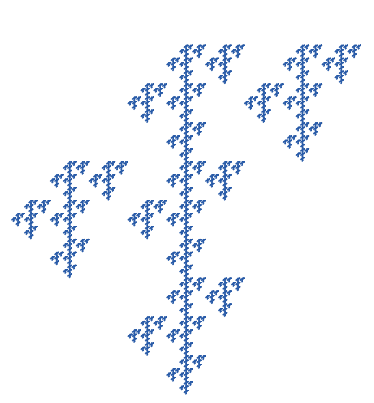

(d)

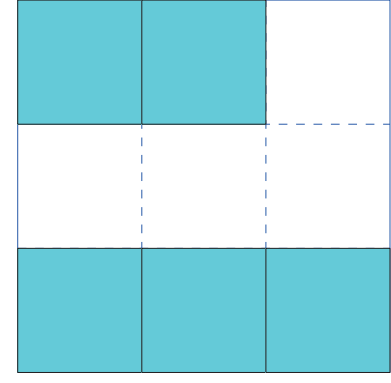

(b)

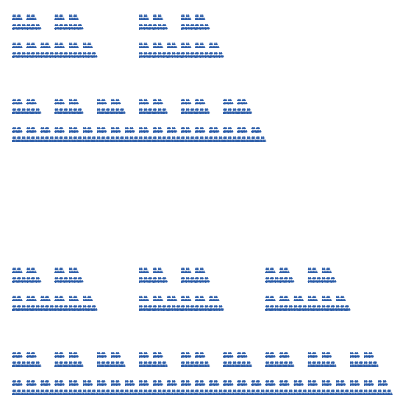

(e)

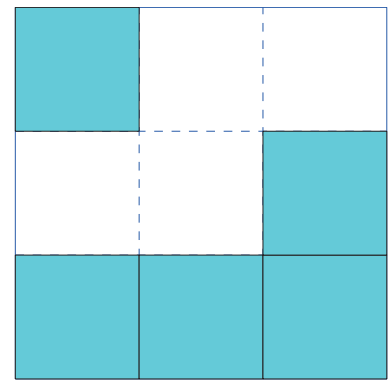

(c)

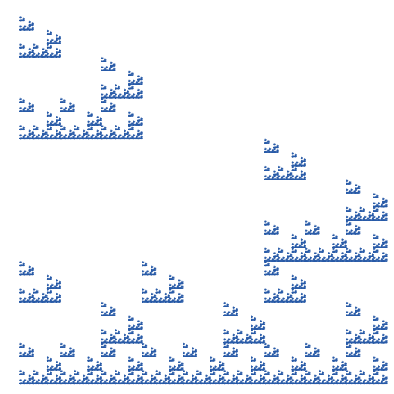

(f)

Figure 3: (d) is equivalent to (e) is proved in [26], (d) is equivalent to (f) is proved in [20].

Let $(M, d)$ be a locally compact, separable metric space, let $v$ be a Radon measure on $M$ with full support. We call $(M, d, v)$ a metric measure space. From now on, we shall always use $|\cdot|$ to denote the Euclidean metric on $\mathbb{R}^{n}$, and use $\mu$ to denote the $\alpha$-dimensional Hausdorff measure. When we write $(F,|\cdot|, \mu)$, we mean that $\mu$ is the restriction of the $\alpha$-dimensional Hausdorff measure on $F$.

For a metric space $(M, d, v), \mathrm{Gu}$ and Lau [8] (see also [7]) define a family of Besov spaces

$$
W^{\sigma, 2} \text { with } \sigma>0 \text {, }
$$




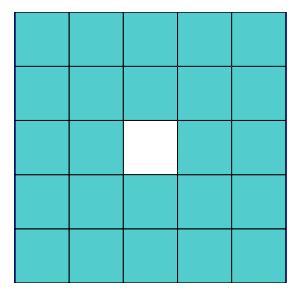

(a)

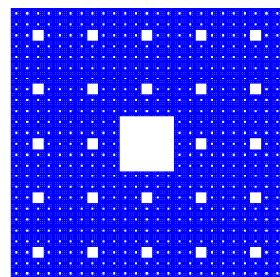

(c)

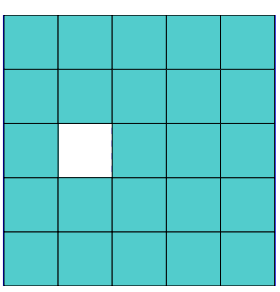

(b)

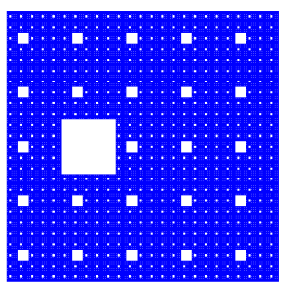

(d)

Figure 4: It is conjectured [21] that the above two fractal squares are not Lipschitz equivalent.

and then define two critical exponents $\sigma^{*}$ and $\sigma^{\#}$ (see Section 2 for precise definition). The main goal of the present paper is to show that both $\sigma^{*}$ and $\sigma^{\#}$ are Lipschitz invariants.

Theorem 1.1. Let $F$ be an $\alpha$-set in $\mathbb{R}^{n}$. Let $T: F \rightarrow T(F)$ be a bi-Lipschitz transform. Denote by $\sigma_{1}^{*}$ and $\sigma_{1}^{\#}$ the critical exponents of $(F,|\cdot|, \mu)$ and by $\sigma_{2}^{*}$ and $\sigma_{2}^{\#}$ the critical exponents of $(T(F),|\cdot|, \mu)$ respectively. Then we have

$$
\sigma_{1}^{*}=\sigma_{2}^{*}, \quad \sigma_{1}^{\#}=\sigma_{2}^{\#} .
$$

Remark 1.1. It is shown [7] that under mild heat kernel assumptions, $\sigma^{*}=\sigma^{\#}$ and the walk dimension $\beta^{*}$ equals to $2 \sigma^{*}$. The walk dimension is an important parameter in the heat kernel estimates, which corresponding to the speed of diffusion on the underlying sets. Heuristically, the larger the value $\beta^{*}$, the harder is for the diffusion process to drift away from the initial position.

The calculation of $\sigma^{*}$ is a difficult problem. It is well-known that for a smooth domain in $\mathbb{R}^{d}, \sigma^{*}=1$; for the $n$-dimensional Sierpinski gasket, $\sigma^{*}=\log (n+3) /(2 \log 2)$ [10]. There are extensions on the nested fractals [16], and approximate value of the Sierpinski carpet [2]; also for Cantor-type sets, $\sigma^{*}=\infty$ [13]. The exact value of $\sigma^{*}$ is only known for limited pcf self-similar sets. Nevertheless, we hope our study may shed some light on the study of Lipschitz equivalence of connected fractals.

The paper is organized as follows. In Section 2, we recall the definition of $\sigma^{*}$ and $\sigma^{\#}$, and prove Theorem 1.1. In Section 3, we discuss the calculation of the critical exponents $\sigma^{*}$ and $\sigma^{\#}$ by showing examples. 
Notation 1.1. Throughout this paper, the sign $f \asymp g$ for two functions $f$ and $g$ means that there exists constant $c>0$ such that $c^{-1} f \leq g \leq c f$. The letters $C, C^{\prime}$ with or without subscripts denote positive constants whose values are not important and may change from line to line.

\section{Critical exponents of Besov spaces and proof of Theorem 1.1}

Let $(M, d, v)$ be a metric measure space. Let $C(M)$ be the continuous function space. For any $1 \leq p \leq \infty$, let $L^{p}(M, v)$ be the Lebesgue function space, and set

$$
\|u\|_{p}=\|u\|_{L^{p}(M, v)} .
$$

For any $\sigma>0$, define the functional $W_{\sigma, M}(u)$ on measurable functions on $M$ by

$$
W_{\sigma, M}(u)=: \sup _{0<r<1} r^{-2 \sigma} \int_{M}\left[\frac{1}{v(B(x, r))} \int_{B(x, r)}|u(x)-u(y)|^{2} d v(y)\right] d v(x),
$$

where $B(x, r)=:\{y \in M: d(x, y)<r\}$ is an open metric ball. Define the space $W^{\sigma, 2}$ as follows,

$$
W^{\sigma, 2}=W^{\sigma, 2}(M, d, v)=:\left\{u \in L^{2}: W_{\sigma, M}(u)<\infty\right\} .
$$

Thus $W^{\sigma, 2}$ is a Banach space with the norm

$$
\|u\|_{\sigma, 2}=:\|u\|_{2}+W_{\sigma, M}(u)^{1 / 2},
$$

and it is one of the families of Besov spaces, see [7, Section 4]. Set

$$
\sigma^{*}=: \sup \left\{\sigma: W^{\sigma, 2} \cap C(M) \text { is dense in } C(M)\right\},
$$

and

$$
\sigma^{\#}=: \sup \left\{\sigma: W^{\sigma, 2} \cap C(M) \text { contains non-constant functions }\right\} .
$$

Clearly we have $\sigma^{*} \leq \sigma^{\#}$. Following [8], we call $\sigma^{*}$ and $\sigma^{\#}$ the critical exponents of the family $W^{\sigma, 2}$ of the Besov spaces in $(M, d, v)$. Note that the critical exponents are intrinsic parameters of $(M, d, v)$.

Before proving Theorem 1.1, we first give two lemmas. Recall that $\mu$ denotes the $\alpha$-dimensional Hausdorff measure.

Lemma 2.1. Assume that all the assumptions in Theorem 1.1 hold. Then for any $1 \leq p \leq \infty$, there exists a constant $\lambda_{p}$ only depending on $F, T$ and $p$ such that for any measurable function $v$ on $F$,

$$
\left\|v \circ T^{-1}\right\|_{L^{p}(T(F))} \leq \lambda_{p}\|v\|_{L^{p}(F)} .
$$


Proof. It is well known that for any Borel set $A \subseteq F$,

$$
C^{-1} \mu(A) \leq \mu(T(A)) \leq C \mu(A)
$$

(see for example, [6]). Therefore, an approximation argument by using simple functions leads to (2.4).

Lemma 2.2. Assume that all the assumptions in Theorem 1.1 hold. Then for any $\sigma>0$, there exist constants $C, C^{\prime}>0$ only depending on $F, T$ and $\sigma$ such that for any $0<r<1$ and any measurable function $u$ on $F$,

$$
\begin{aligned}
& \int_{T(F)}\left[\int_{B(x, r)}\left|u \circ T^{-1}(x)-u \circ T^{-1}(y)\right|^{2} d \mu(y)\right] d \mu(x) \\
\leq & C^{\prime} \int_{F}\left[\int_{B(x, C r)}|u(x)-u(y)|^{2} d \mu(y)\right] d \mu(x) .
\end{aligned}
$$

Proof. Fix $x \in T(F)$, then by using the fact that $T$ is Lipschitz, there exists $C>0$ which is independent of $x$ such that

$$
T^{-1}(B(x, r)) \subseteq B\left(T^{-1} x, C r\right) .
$$

Applying Lemma 2.1 to the $L^{2}$ function

$$
v \circ T^{-1}(y)=\left|u \circ T^{-1}(x)-u \circ T^{-1}(y)\right| \cdot 1_{B(x, r)}(y),
$$

we obtain

$$
\begin{aligned}
& \int_{B(x, r)}\left|u \circ T^{-1}(x)-u \circ T^{-1}(y)\right|^{2} d \mu(y) \\
\leq & \lambda_{2} \int_{T^{-1}(B(x, r))}\left|u \circ T^{-1}(x)-u(z)\right|^{2} d \mu(z) \\
\leq & \lambda_{2} \int_{B\left(T^{-1} x, C r\right)}\left|u \circ T^{-1}(x)-u(z)\right|^{2} d \mu(z) .
\end{aligned}
$$

Thus by integrating on $x$ over $T(F)$, we get

$$
\begin{aligned}
& \int_{T(F)}\left[\int_{B(x, r)}\left|u \circ T^{-1}(x)-u \circ T^{-1}(y)\right|^{2} d \mu(y)\right] d \mu(x) \\
\leq & \lambda_{2} \int_{T(F)}\left[\int_{B\left(T^{-1} x, C r\right)}\left|u \circ T^{-1}(x)-u(z)\right|^{2} d \mu(z)\right] d \mu(x) .
\end{aligned}
$$

Applying Lemma 2.1 again to the $L^{1}$ function

$$
v \circ T^{-1}(x)=\int_{B\left(T^{-1}(x), C r\right)}\left|u \circ T^{-1}(x)-u(z)\right|^{2} d \mu(z),
$$


we have

$$
\begin{aligned}
& \int_{T(F)}\left[\int_{B\left(T^{-1} x, C r\right)}\left|u \circ T^{-1}(x)-u(z)\right|^{2} d \mu(z)\right] d \mu(x) \\
\leq & \lambda_{1} \int_{F}\left[\int_{B(x, C r)}|u(x)-u(y)|^{2} d \mu(y)\right] d \mu(x) .
\end{aligned}
$$

Combining (2.8) and (2.9), we have (2.6) with $C^{\prime}=\lambda_{1} \lambda_{2}$.

Proof of Theorem 1.1. We only need to show that $\sigma_{1}^{*} \leq \sigma_{2}^{*}$ and $\sigma_{1}^{\#} \leq \sigma_{2}^{\#}$ separately. In the following, we simply write $W^{\sigma, 2}(F)$ instead of $W^{\sigma, 2}(\bar{F},|\cdot|, \mu)$ without causing confusion and similar for $W^{\sigma, 2}(T(F))$.

Case for $\sigma^{\#}$. For any $\sigma<\sigma_{1}^{\#}$, by definition we can find a non-constant function $u \in$ $W^{\sigma, 2}(F)$, thus $\|u\|_{L^{2}(F)}<\infty$ and $W_{\sigma, F}(u)<\infty$.

Firstly, by Lemma 2.1, we have $u \circ T^{-1} \in L^{2}(T(F))$. Secondly, by Lemma 2.2, we have

$$
\begin{aligned}
& W_{\sigma, T(F)}\left(u \circ T^{-1}\right) \\
\leq & \sup _{0<r<1} C_{1} r^{-2 \sigma-\alpha} \int_{T(F)}\left[\int_{B(x, r)}\left|u \circ T^{-1}(x)-u \circ T^{-1}(y)\right|^{2} d \mu(y)\right] d \mu(x) \\
\leq & C^{\prime} \sup _{0<r<1} r^{-2 \sigma-\alpha} \int_{F}\left[\int_{B(x, C r)}|u(x)-u(y)|^{2} d \mu(y)\right] d \mu(x) \\
\leq & C^{\prime} \sup _{0<r<C} r^{-2 \sigma-\alpha} \int_{F}\left[\int_{B(x, r)}|u(x)-u(y)|^{2} d \mu(y)\right] d \mu(x) \\
\leq & C^{\prime}\left\{\sup _{0<r<1}+\sup _{1 \leq r<C}\right\} r^{-2 \sigma-\alpha} \int_{F}\left[\int_{B(x, r)}|u(x)-u(y)|^{2} d \mu(y)\right] d \mu(x) \\
\leq & C^{\prime} W_{\sigma, F}(u)+2 C^{\prime} \int_{F}|u(x)|^{2} \sup _{x \in F} \mu(B(x, C)) \\
\leq & C^{\prime} W_{\sigma, F}(u)+C^{\prime} C^{\alpha}|| u \|_{L^{2}(F)}<\infty .
\end{aligned}
$$

Therefore $u \circ T^{-1}$ is a non-constant function in $W^{\sigma, 2}(T(F))$, which implies that $\sigma \leq \sigma_{2}^{\#}$. Since $\sigma<\sigma_{1}^{\#}$ is assumed arbitrary, we conclude that $\sigma_{1}^{\#} \leq \sigma_{2}^{\#}$.

Case for $\sigma^{*}$. For any $\sigma<\sigma_{1}^{*}$, by definition we can find a sequence of functions $\left\{u_{n}\right\}_{n=1}^{\infty}$ in $W^{\sigma, 2}(F) \cap C(F)$ such that it is dense in $C(F)$. Now pick any $v \in C(T(F))$, we have $v \circ T \in C(F)$ and there is a subsequence of $\left\{u_{n}\right\}_{n=1}^{\infty}$ which we still denote by $\left\{u_{n}\right\}_{n=1}^{\infty}$ such that $u_{n} \rightarrow v \circ T$ in $C(F)$ as $n \rightarrow \infty$. We then clearly have $u_{n} \circ T^{-1} \rightarrow v$ in $C(T(F))$ as $n \rightarrow \infty$ by that $T$ is bi-Lipschitz. On the other hand, from the argument we used in the first case, we have $u_{n} \circ T^{-1} \in W^{\sigma, 2}(T(F)) \cap C(T(F))$. This implies that $\left\{u_{n} \circ T^{-1}\right\}_{n=1}^{\infty}$ is dense in $C(T(F))$, and thus $\sigma \leq \sigma_{2}^{*}$. Consequently, by that $\sigma<\sigma_{1}^{*}$ is arbitrary we have $\sigma_{1}^{*} \leq \sigma_{2}^{*}$. 


\section{Examples}

In this section, we are concerned with the calculation of $\sigma^{*}$ and $\sigma^{\#}$ for $\alpha$-sets. In the case that the metric space $(M, d, v)$ admits a heat kernel with certain fast decay condition (e.g., sub-Gaussian), we know from [7] that

$$
\beta=2 \sigma^{*}=2 \sigma^{\#},
$$

where $\beta$ is the walk dimension of the heat kernel.

Theorem 3.1 (Theorem 4.6 in [7]). If there exists a heat kernel $p_{t}$ on $(M, d, v)$ satisfying the following decay condition:

$$
\begin{aligned}
& p_{t}(x, y) \asymp \frac{C}{t^{\alpha / \beta}} \Phi\left(c \frac{d(x, y)}{t^{1 / \beta}}\right) . \\
& \int_{0}^{\infty} s^{\alpha+\beta+\varepsilon} \Phi(s) \frac{d s}{s}<\infty,
\end{aligned}
$$

where $0<t<t_{0}$ with $t_{0}>0$ and $\Phi$ is a decreasing function which maps $[0,+\infty)$ onto $(0,1]$. Then $\beta=\beta^{*}$ (the number $\beta$ satisfying (3.1a) and (3.1b) is called the walk dimension of $M$ ).

Another fact from [7] is that provided the space $(M, d, v)$ ( $v$ is an $\alpha$-regular measure) having the chain condition, the following inequality holds:

$$
1 \leq \sigma^{*} \leq \sigma^{\#} \leq(\alpha+1) / 2
$$

In general, see the following definition of the $\gamma$-chain condition.

Definition 3.1. Fix $\gamma>0$, a metric space $(M, d)$ is said to satisfy the $\gamma$-chain condition if there exists a constant $C>0$ such that for any two points $p, q \in M$ and for any positive integer $n$ there exists a sequence $\left\{q_{i}\right\}_{i=0}^{n}$ of points in $M$ such that $q_{0}=p, q_{n}=q$ and

$$
d\left(q_{i}, q_{i+1}\right) \leq C \frac{d(p, q)}{n^{\gamma}} \quad \text { for all } i=0,1, \cdots, n-1 .
$$

Remark 3.1. The chain condition is the special case when $\gamma=1$, also it is not hard to see that the $\gamma$-chain condition as a geometric property is Lipschitz invariant, i.e., it is preserved under bi-Lipschitz maps of two metric spaces.

Note that certain bounds of heat kernel is equivalent to the so called $\beta$-parabolic Harnack principle (see [3, Theorem 2.15] for details). It is shown in [3, Theorem 2.21] that the $\beta$-parabolic Harnack principle is preserved for metric measure spaces or weight graphs under rough isometries. 

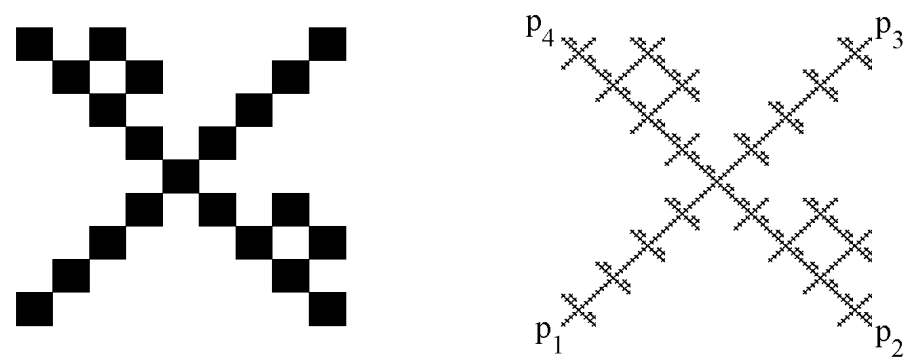

Figure 5: The eyebolted Vicsek cross $K$.

Definition 3.2. A map $\varphi: X_{1} \rightarrow X_{2}$ is called a rough isometry between two metric measure spaces or weight graphs $\left(X_{i}, d_{i}, \mu_{i}\right)$ for $i=1,2$ if there are positive constants $c_{1}$, $c_{2}, c_{3}$ such that

$$
\begin{aligned}
& X_{2}=\bigcup_{x \in X_{1}} B_{d_{2}}\left(\varphi(x), c_{1}\right), \\
& c_{2}^{-1}\left(d_{1}(x, y)-c_{1}\right) \leq d_{2}(\varphi(x), \varphi(y)) \leq c_{2}\left(d_{1}(x, y)+c_{1}\right), \\
& c_{3}^{-1} \mu\left(B_{d_{1}}\left(x, c_{1}\right)\right) \leq \mu\left(B_{d_{2}}\left(\varphi(x), c_{1}\right) \leq c_{3} \mu\left(B_{d_{1}}\left(x, c_{1}\right)\right) .\right.
\end{aligned}
$$

It is seen that rough isometry is an equivalence and bi-Lipschitz will imply rough isometry. Hence in the case that the $\beta$-parabolic Harnack principle or equivalently certain bounds of heat kernels are assumed on $\left(X_{1}, d_{1}, \mu_{1}\right)$, then the $\beta$-parabolic Harnack principle is true on its rough isometric space $\left(X_{2}, d_{2}, \mu_{2}\right)$ and this implies our result in the case that heat kernel estimates are assumed. However, we will show that there are examples not included in this setup. The examples are from [8], we refer to [8, Theorem 4.1,Theorem 4.3] in which the criteria are given to determine the values of $\sigma^{*}$ and $\sigma^{\#}$ in terms of the growth ratios of resistances with respect to primal energies for a class of pcf self-similar sets.

Example 3.1 (Eyebolted Vicsek cross). In $\mathbb{R}^{2}$, let $\left\{p_{1}, p_{2}, p_{3}, p_{4}\right\}$ be the four vertices of the unit square $S$, and let $p_{0}$ be the center of $S$. Divide $S$ into a mesh of sub-squares of size 1/9, and we pick 21 sub-squares in two different patterns as shown in Fig. 5 and Fig. 6 separately.

Let $K$ be corresponding self-similar set in Fig. 5 and $G$ be in Fig. 6 . The Hausdorff dimensions of $K$ and $G$ are the same, that is $\alpha=\log 21 / \log 9$.

It is known [8, Theorem 1.2] that for $K$,

$$
\sigma^{*}=\sigma^{\#}=\frac{1}{2}\left(1+\frac{\log 21}{\log 9}\right) .
$$

Also it is known that the Besov space at $\sigma^{*}, W^{\sigma^{*}, 2}$ is not dense in $C(K)$, so it can not be a candidate of the domain of some regular Dirichlet forms and hence there does not 

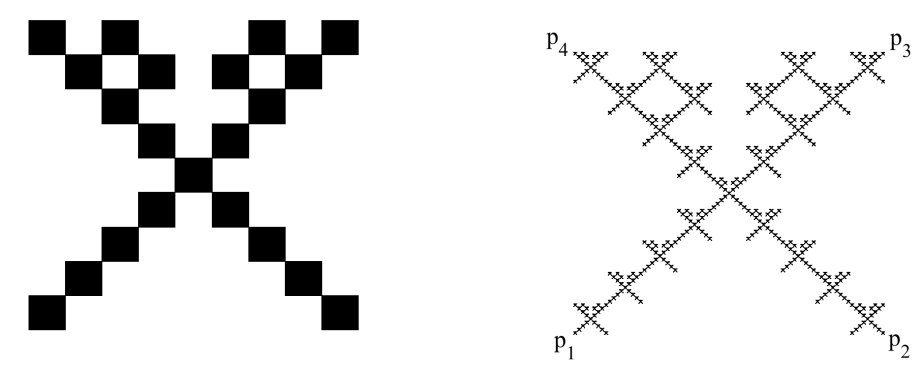

Figure 6: The eyebolted Vicsek cross G.

exist a sub Gaussian heat kernel with walk dimension $\beta^{*}$ or its equivalent condition the $\beta^{*}$-parabolic Harnack principle.

On the other hand, we see from [8, Section 7] that for $G$,

$$
\sigma^{*}=\sigma^{\#}=\frac{1}{2}\left(\frac{\log 21+\log (35 / 4)}{\log 9}\right) .
$$

Moreover, the Besov space $W^{\sigma^{*}, 2}$ is a domain of some regular Dirichlet form which has a sub-Gaussian heat kernel with $\beta^{*}=2 \sigma^{*}$ as its walk dimension. The methods to obtain the sub-Gaussian heat kernel estimates are standard (e.g., $[1,9,12,14])$, for instance, first deducing Nash-type inequality to obtain the existence and on-diagonal upper bound of the heat kernel, and together with the estimation of the exist time from balls, one can obtain the off-diagonal upper bound; Using the upper bound to get a near-diagonal lower bound, and together with a chain argument (the Euclidean metric on $G$ satisfies the chain condition), one can obtain the off-diagonal lower bound.

From above, by using Theorem 1.1, we see that $K$ and $G$ are not Lipschitz equivalent since they have different critical exponents of Besov spaces.

\section{Acknowledgements}

The second author is supported by NSFC Nos. 10631040 and 11471075.

\section{References}

[1] M. Barlow, Diffusions on Fractals, vol. 1690 of Lecture Notes Math., Springer, 1998, 1-121.

[2] M. Barlow and R. Bass, Transition densities for Brownian motion on the Sierpiński carpet, Probab. Theory Related Fields, 91 (1992), 307-330.

[3] M. Barlow, R. Bass and T. Kumagai, Stability of parabolic Harnack inequalities on metric measure spaces, J. Math. Soc. Japan, 58 (2006), 485-519.

[4] G. David and S. Semmes, Fractured Fractals and Broken Dreams : Self-Similar Geometry through Metric and Measure, Oxford University Press, 1997. 
[5] K. J. Falconer and D. T. Marsh, On the Lipschitz equivalence of Cantor sets, Mathematika, 39 (1992), 223-233.

[6] K. J. Falconer, Fractal Geometry, Mathematical Foundations and Applications, Wiley, New York, 1990.

[7] A. Grigor'yan, J. Hu, and K.-S. Lau, Heat kernels on metric-measure spaces and an application to semilinear elliptic equations, Trans. Amer. Math. Soc., 355 (2003), 2065-2095.

[8] Q. Gu and K.-S. Lau, Dirichlet forms and critical exponents on fractals, Trans. Amer. Math. Soc., (to appear).

[9] J. Hu, An analytical approach to heat kernel estimates on strongly recurrent metric spaces, Proc. Edin. Math. Soc., 51 (2008), 171-199.

[10] A. Jonsson, Brownian motion on fractals and function spaces, Math. Zeit., 222 (1996), 495504.

[11] A. Jonsson and H. Wallin, Function spaces on subsets of $R^{n}$, Math. Reports, Vol. 2, Acad. Publ., Harwood, 1984.

[12] J. Kigami, Harmonic analysis for resistance forms, J. Func. Anal., 204 (2003), 399-444.

[13] S.-L. Kong, K.-S. Lau and T.-K. Wong, Random walks and induced Dirichlet forms on selfsimilar sets, Adv. Math., 320 (2017), 1099-1134.

[14] T. Kumagai, Estimates of the transition densities for Brownian motion on nested fractals, Probab. Theory and Related Fields, 96 (1993), 205-224.

[15] J. J. Luo and K.-S. Lau, Lipschitz equivalence of self-similar sets and hyperbolic boundaries, Adv. Math., 235 (2013), 555-579.

[16] K. Pietruska-Pałuba, Some function spaces related to the Brownian motion on simple nested fractals, Stochastics and Stochastics Reports, 67 (1999), 267-285.

[17] H. Rao, H. J. Ruan and Y. Wang, Lipschitz equivalence of self-similar sets: algebraic and geometric properites, Contemp. Math., 600 (2013).

[18] H. Rao, H. J. Ruan and L. F. Xi, Lipschitz equivalence of self-similar sets, C. R. Acad. Math. Sci. Paris., 342 (2006), 191-196.

[19] H. Rao and Y. Zhang, Higher dimensional Frobenius problem: Maximal saturated cone, growth function and rigidity, J. Math. Pures Appl., 104 (2015), 868-881.

[20] H. Rao and Y. J. Zhu, Lipschitz equivalence of fractal squares which are not totally disconnected, preprint, 2016.

[21] H. J. Ruan and Y. Wang, Topological invariants and Lipschitz equivalence of fractal squares, preprint, 2015.

[22] H. J. Ruan, Y. Wang, and L. F. Xi, Lipschitz equivalence of self-similar sets with touching structures, Nonlinearity, 27 (2014), 1299-1321.

[23] G. T. Whyburn, Topological characterization of the Sierpinski curve, Fund. Math., 45 (1958), 320-324.

[24] L. F. Xi and Y. Xiong, Self-similar sets with initial cubic patterns, C. R. Math. Acad. Sci. Paris, 348 (2010), 15-20.

[25] L. F. Xi and Y. Xiong, Lipschitz equivalence of fractals generated by nested cubes, Math. Zeit., 271 (2012), 1287-1308.

[26] Y. J. Zhu and Y. M. Yang, Lipschitz equivalence of self-similar sets with two-state neighbor automaton, J. Math. Anal. Appl., 458 (2018), 379-392. 